\title{
L'ADOZIONE DEL PENSIERO SISTEMICO NELLA GESTIONE PUBBLICA: UN CAMBIAMENTO DI PARADIGMA
}

\section{RECENSIONE ARTICOLO}

REBELO, Michele Alves Correa ${ }^{1}$

REBELO, Michele Alves Correa. L'adozione del pensiero sistemico nella gestione pubblica: un cambiamento di paradigma. Revista Científica Multidisciplinar Núcleo do Conhecimento. anno 04, Ed. 10, Vol. 01, pp. 45-63. nell'ottobre 2019. ISSN: 24480959, Collegamento accesso: https://www.nucleodoconhecimento.com.br/economia-aziendale/pensierosistemico

\section{RIEPILOGO}

Considerato l'attuale scenario politico brasiliano e i cambiamenti nel panorama della gestione pubblica nel XXI secolo, questo articolo mira a presentare le riflessioni dell'adozione del pensiero sistemico da parte del direttore pubblico che evita l'influenza che questo pensiero Decisionale. È dedotta da una ricerca di natura applicata, di un obiettivo esplorativo, con l'adozione della ricerca bibliografica. Si tratta di una questione importante, dovuta alla difficoltà del gestore pubblico di raggiungere l'efficienza nell'esecuzione delle attività amministrative. Con la ricerca è stato possibile osservare che il pensiero sistemico del gestore influenza la direzione della gestione pubblica, i benefici di questa posizione possono essere visibili nei servizi forniti alla società e alle azioni interne dell'istituzione. Al giorno d'oggi, si osserva la personalità nel processo decisionale, a causa dell'influenza politica dello Stato e perché è uno scenario altamente competitivo, dove prevalgono la vanità personale e la cultura dell'immediatezza. II pensiero sistemico contribuisce a nuove visioni emergenti che

\footnotetext{
${ }^{1}$ Master nella gestione delle politiche pubbliche - UNIVALI. Specialista in diritto penale e procedurale-scuola della magistratura di Santa Catarina. Specialista nella gestione della sicurezza pubblica - UNIVALI. Laurea in Giurisprudenza - FEBE.
} 
diventano condivise e collaborative. La gestione pubblica manca di un'innovazione sistematica del pensiero per soddisfare le esigenze sociali e raggiungere l'efficienza.

Parole chiave: gestione pubblica, responsabile pubblico, principio di efficienza, pensiero sistemico.

\section{INTRODUZIONE}

La presente ricerca propone di dimostrare ai lettori le riflessioni sull'adozione del pensiero sistemico da parte del direttore pubblico e come ciò possa influenzare la vita delle persone.

Dal decennio degli anni ' 80 , è stato osservato un movimento in crescita alla ricerca di tecniche di gestione moderne ed efficienti, in grado di fornire miglioramenti nei servizi pubblici (KETTL, 2005; PAULA, 2005; MANNING et al., 2009). Parallelamente a questa realtà, il direttore pubblico si è imbattuto in un nuovo ambiente globale, nuove esigenze sociali, nuove tecnologie e concetti di progetto.

A causa di questi fattori, il modo di amministrare i servizi pubblici si è trasformato. II modello burocratico viene sostituito dal modo manageriale e i cambiamenti politici, sociali e culturali cominciano a interferire nel modo di pensare della società.

Nonostante questo progresso nei concetti di gestione e pubblica amministrazione, si percepisce la difficoltà del gestore pubblico di raggiungere l'efficienza nell'esecuzione delle sue attività. Ciò è dovuto alla priorità degli interessi particolari dei candidati alle posizioni di direzione, leadership e consulenza, nonché all'influenza politica sul processo decisionale dei dirigenti pubblici.

Secondo il consiglio di amministrazione (2009), la funzione di direzione, resa possibile dall'accesso alla posizione pubblica classificata come libera disposizione, implica le aspettative dell'esecuzione della posizione associata agli interessi politici di coloro che hanno il potere di nominare. Per questo motivo, è richiesta fedeltà personale al nominato. Questo è un legame precario e può essere esonerato in qualsiasi momento. 
Nella tradizione amministrativa brasiliana, solo il nucleo più tecnico dello Stato tende ad avere posizioni di alto livello conservate da nomine marcatamente politiche (FLEURY, 2009). Idealmente, queste posizioni sarebbero occupate da persone che avevano conoscenze tecniche. Tuttavia, è adottato nella maggior parte dei casi, criterio di denominazione soggettiva.

L'obiettivo di questo lavoro è quello di cercare una riflessione su una nuova posizione del pubblico manager di fronte a questo problema. Sarà necessario affrontare in modo superficiale aspetti di natura soggettiva, come il carattere del gestore, che determina il comportamento morale o sociale e, di conseguenza, la direzione di una buona o cattiva gestione.

La gestione pubblica manca di nuove posizioni di potere e di leadership. Un'innovazione sistemica del pensiero dovrebbe avvenire in qualsiasi momento dell'istituzione. Non si tratta solo di fare cose diverse, ma di fare le stesse cose in modi diversi, creando, come cita Peter Drucker (2002), nuovi potenziali di soddisfazione.

Data la complessità del tema e da un'analisi sistematica, abbiamo il diritto e il gestore come sistemi aperti, che si riferiscono in una messa a punto dinamica. Sono in continuo e incessante processo di scambi e scambi con l'ambiente. Le organizzazioni sono sistemi di ruoli svolti dalle persone (CHIAVENATO, 2000).

Sotto la visione e il contributo delle scienze sociali, vi è la possibilità di condurre la ricerca basata sulla Teoria di Bertalanffy (1937), che ha una visione diversa del riduzionismo scientifico fino ad allora applicato dalla scienza convenzionale.

Secondo Valena (2011), l'approccio sistemico è direttamente associato alla lettura della complessità del ragionamento, della padronanza o della supervenienza delle emozioni nelle decisioni umane e negli stimoli a una decisione efficace.

Pensare sistematicamente è pensare alla complessità, all'instabilità e all'intersoggettività. Un professionista che vive - vede il mondo e agisce in esso - le implicazioni di aver assunto le ipotesi di cui sopra, possono essere considerate un 
professionista sistemico. Deve avere lo sguardo dell'osservatore - l'Observer come parte del sistema (VASCONCELOS, 2009).

Con il tema delineato, si propone di dimostrare le riflessioni dell'adozione del pensiero sistemico e le influenze che questa postura può generare nella vita delle persone, rispondendo alla seguente domanda: sarà possibile raggiungere l'efficienza con l'adozione del pensiero sistemico Un manager pubblico?

Per raggiungere l'obiettivo proposto, il metodo descrittivo sarà utilizzato attraverso la ricerca bibliografica.

Per Fonseca (2002), la ricerca bibliografica è fatta dall'indagine sui riferimenti teorici già analizzati e pubblicata con mezzi scritti ed elettronici, come libri, articoli scientifici e pagine di siti web.

Nel prossimo capitolo sarà presentato il concetto di gestione pubblica e, in punto specifico, il manager. II principio di efficienza sarà quindi affrontato in modo superficiale. E infine, presenteremo il pensiero sistemico e le riflessioni su questo sguardo nella costruzione di un nuovo paradigma di gestione pubblica.

\section{GESTIONE PUBBLICA}

Negli ultimi 30 anni il termine "gestione pubblica" è stato utilizzato in sostituzione con la pubblica amministrazione e, per questo motivo, è stato considerato un termine polisemico, poiché una parte della dottrina capisce che i due termini sono sbagliati. Altri sostengono che c'è stata una rottura del concetto tradizionale, perché comprende strumenti di gestione del mondo degli affari e, quindi, un concetto più ampio.

Henri Fayol (1950), già nel decennio dei 50 anni, definì la gestione come funzioni amministrative di previsione, organizzazione, comando, coordinamento e controllo.

Dal decennio degli 80 anni, i governi di diversi paesi hanno intrapreso sforzi per modernizzare la pubblica amministrazione al fine di ridurre la portata del governo e farla funzionare meglio nel perseguimento dell'agilità. La valutazione della pubblica 
amministrazione stessa cambia e viene quindi smizzata da criteri vicini a quelli utilizzati dall'amministrazione privata.

Come risultato di questa posizione nasce il New Public Manegement (NPM), associato al contesto di una specifica riforma dello Stato, il cui modello si oppone alla pubblica amministrazione convenzionale, sulla base del modello di burocrazia weberianeinjured consiste nel conferire un Approccio manageriale, ispirato ai metodi della gestione aziendale privata, alla pubblica amministrazione (HOOD, 1995; HERNES, 2005), al fine di garantire una maggiore reattività e migliori prestazioni nella fornitura di servizi pubblici alla popolazione (MANNING et al., 2009).

Perry e Kraemer (1983) Ritengono che la gestione pubblica sia una fusione dell'orientamento normativo della pubblica amministrazione tradizionale e della guida strumentale della direzione, in senso generico. In altre parole, nel primo aspetto, la gestione pubblica incorpora temi come la democrazia e la responsabilità, e valori come l'equità, l'uguaglianza e la probità. E nel secondo, un orientamento strumentale, con l'idea che il settore pubblico condivide con il privato la necessità di raggiungere i suoi obiettivi in modo più economico ed efficiente.

Per Druker (1993) la direzione non si riferisce alla gerarchia organizzativa di un'amministrazione classica, ma alla capacità di promuovere l'innovazione sistematica della conoscenza e di trarne la massima resa nella sua applicazione alla produzione.

A differenza della gestione privata, la gestione pubblica deve essere guidata da valori sociali. Si presume qui, la concezione che la gestione è un atto complesso che ci avvicina al mondo della politica (BRUGU; SUBIRATS, 1996).

Pensare all'evoluzione storica della gestione pubblica, prospetticamente, si riferisce a una riflessione sul vero ruolo dello Stato di oggi. Gestire ciò che è pubblico è un atto complesso e impegnativo perché lo scenario è altamente competitivo. La vanità personale e la cultura dell'immediatezza prevalgono in questo mezzo. Ogni legislatura viene modificata la dinamica dell'amministrazione. Non c'è continuità delle azioni a 
causa della personalità nel processo decisionale, in considerazione dell'influenza politica che entusiasma il paese.

La gente è terrorizzata dalla notizia di crimini barbarici che vengono truffati dai media. Tuttavia, non si rendono conto che un lavoro troppo caro o incompiuto, a causa di cattiva gestione, può aver contribuito al caos nell'istruzione e nella salute di una determinata regione.

Cardoso Jr (2001) sostiene che la responsabilità dello Stato non è solo quella di fare le cose meglio e in modo più efficiente. Più di questo, è compito di indurre, promuovere o addirittura produrre le condizioni per trasformare le strutture economiche e sociali del paese. II processo prevede la riprogettazione della struttura, delle procedure e delle pratiche del settore pubblico, incorporando cambiamenti di grande portata nella dimensione istituzionale, che comprendono questioni legate ai principi e ai valori che informano il rapporto di tali organizzazioni Con la società e il mercato (FERLIE et al., 1999; Ormond LOFFLER, 1999; HERNES, 2005).

In questo senso, la gestione pubblica manca di innovazione sistemica per soddisfare le esigenze sociali e raggiungere l'efficienza. L'adozione del pensiero sistemico da parte del manager può contribuire a nuove visioni emergenti che diventano visioni condivise e collaborative. La visione del tutto è fondamentale per il processo decisionale.

Per Abràcio (2007), la gestione pubblica ha una serie di peculiarità che riguardano la necessità di disporre di strumenti manageriali e democratici per combattere i problemi che lo Stato si trova ad affrontare nel mondo contemporaneo. In questo contesto, il formalismo e la rigidità burocratica dovrebbero essere attaccati come mali, e la meritocrazia può promuovere la modernizzazione.

La visione condivisa è il primo passo per far in modo che le persone che non si fidano I'una dell'altra inizino a lavorare insieme. Crea un'identità comune. (SENGE,2009). Attraverso una cultura del feedback, il rapporto di fiducia nel team e il riconoscimento 
dello sforzo contribuiscono all'efficienza della gestione pubblica, in quanto raggiunge l'aspetto legato alla motivazione.

II manager può trasformare i concetti (pre) stabiliti nel corso della storia. Nonostante sia legato al principio di legalità, puoi fare la differenza. Per questo, deve assumere una nuova postura, lo sguardo sistemico nel perseguimento dell'efficienza, i cui concetti e aspetti saranno discussi di seguito.

\subsection{DIRETTORE PUBBLICO}

Secondo il concetto classico sviluppato da Henri Fayol (1950), il manager è definito dalle sue funzioni nell'organizzazione. È la persona che è responsabile dell'interpretazione degli obiettivi proposti dall'istituzione e agisce attraverso la pianificazione al fine di raggiungere gli obiettivi e gli obiettivi.

Si può quindi dire che il manager è qualcuno che sviluppa il piano strategico e operativo, giudica i mezzi efficaci, concepisce le strutture e stabilisce le norme e le procedure politiche più appropriate $e$, in ultima analisi, implementa e coordina l'attuazione dei progetti Al centro di un particolare tipo di comando (o leadership).

Per Chiavenato (2004) essere un manager o un leader è avere una visione globale, un rapporto tra l'uomo e il suo ambiente di lavoro. Inoltre, è imparare a insegnare e imparare, essendo il secondo di vitale importanza. Iniziando per una premessa più soggettiva, Peter Drucker (1993) afferma che è attraverso il personaggio che viene esercitato la leadership. Nella stessa linea di pensiero, Havard (2011) definisce che la virtù, oltre ad essere un valore intrinseco dell'essere umano, è una forza dinamica che aumenta la capacità di azione, una caratteristica che è così necessaria al leader. La virtù crea fiducia, e senza fiducia diventa impossibile guidare.

Essere un manager pubblico è una sfida, perché gestire qualcosa che è pubblico è un atto complesso a causa del panorama politico altamente competitivo. Tenuto conto dei frequenti titoli dei media di corruzione, deviazioni dai fondi pubblici, è stato stabilito un paradigma che i servizi pubblici non servono la società in modo efficiente. 
Ad ogni legislatura, la dinamica dell'amministrazione viene alterata, con cambiamenti radicali che si verificano. Questo processo è in corso, perché altrimenti ci sarebbe la dittatura o la monarchia. Tuttavia, è necessaria una continuità di azioni e progetti. È molto importante che il manager abbia la scienza e la nozione di quanto sia importante cambiare la postura nell'esecuzione delle attività quotidiane e come questo atteggiamento possa trasformare la vita delle persone. Una decisione saggia può significare l'educazione dei bambini bisognosi in una determinata regione. Più che mai, la società grida per risultati diversi. È necessario rivedere il processo tradizionale di pensiero pubblico e di attuazione di nuovi atteggiamenti.

II direttore pubblico come nell'iniziativa privata ha bisogno delle caratteristiche e delle qualità che gli consentono di promuovere i cambiamenti proposti. In quanto leader nel settore pubblico, i dirigenti devono essere dotati di conoscenze, competenze e atteggiamenti, nonché di una forte vocazione ad affrontare le varie forme e fonti di potere che permeano il settore pubblico (MORGAN, 1996).

L'adozione del pensiero sistemico è intimamente legata allo sviluppo delle virtù etiche del manager e/o del leader. Possiamo mettere in relazione alcune caratteristiche con gli aspetti sistemici, vale a dire: leadership focalizzata sulla cooperazione e l'influazione, comunicabilità con enfasi sulle relazioni, l'umanitarismo con enfasi sul processo, il pensiero di rete, la flessibilità, Visione ampia con totalità e dinamismo (ANDRADE, 2016).

L'azione di somministrazione è legata alla pratica del pensiero sistemico e della leva strategica. Le caratteristiche della leadership, nel contesto dell'interazione umana che favorisce i processi di ottimizzazione dell'apprendimento, sono orientate all'esercizio della partecipazione a tutti i livelli e alla costruzione di squadre.

L'idea dell'inefficacia dei servizi pubblici dovrebbe essere abbandonata e l'influenza politica ridotta. È necessario comprendere che se l'attenzione si concentra su una parte isolata finalizzata a interessi limitati, non sarà possibile percepire l'intero sistema, essendo fondamentale pensare al tutto e non nelle fasi, se si vuole correggere le 
direzioni e raggiungere l'efficienza, la cui tematica verrà visualizzato nell'argomento seguente.

\section{PRINCIPIO DI EFFICIENZA}

Inizialmente, è necessario stabilire che i principi amministrativi sono orientamenti di base che comprimono e sostengono il modo di gestire gli agenti pubblici, quando le attribuzioni legate alla pubblica amministrazione sono efficaci.

Il principio di efficienza, previsto nell'arte. 37 della CF del 1988, ha avuto origine nel diritto privato, essendo inserito nella pubblica amministrazione, nella riforma amministrativa.

L'efficienza nel settore pubblico è un requisito della nuova tendenza globale a soddisfare gli interessi collettivi in modo rapido e con risultati efficaci. Cerca uno stato che pianifica, sviluppa e svolge le sue funzioni in modo efficace, facendo uso di nuove tecniche e abitudini che mirano a risultati soddisfacenti.

Il principio di efficienza può essere inteso in due pregiudizi: in primo luogo in relazione al modo di azione dell'agente pubblico, che ci si aspetta la migliore performance possibile delle sue attribuzioni, mirando ai migliori risultati e, in secondo luogo, in relazione al modo di organizzare, Strutturare e disciplinare la pubblica amministrazione, anche con l'obiettivo di ottenere i migliori risultati nella fornitura di servizio pubblico (DI PIETRO, 2014).

L'attività amministrativa deve essere condotta da dirigenti impegnati e riguardalto l'efficacia nella fornitura di servizi pubblici. La caratteristica principale del manager dovrebbe essere il pensiero sistemico, come un modo per visualizzare l'intero processo. Una gestione efficiente ha lo sviluppo sociale come filone. Pertanto, l'obiettivo principale del manager dovrebbe essere legato all'uso dei migliori mezzi per raggiungere la soddisfazione delle esigenze collettive. 
Il cambio di posizione è legato alla nuova visione dei dirigenti e dei cittadini in relazione all'amministrazione della cosa pubblica. È necessario staccare dall'opinione obsoleta che l'interesse dello Stato si sovrapponga all'interesse privato.

II costituzionalismo moderno non solo ha posizionato l'uomo all'epicentro dell'ordinamento giuridico, garantendo una protezione differenziata dei diritti fondamentali individuali, ma anche interessi istruiti di natura collettiva, che superano la sfera individuale, in Lo scopo di consentire il godimento dei diritti da parte di tutti i membri della comunità politica (BINENBOJM,2010).

Il termine interesse pubblico deve essere interpretato come la massima realizzazione di tutti gli interessi, individuali e collettivi, legalmente protetti. Nell'attuale stato democratico di diritto, i diritti fondamentali della società si sovrappongono e la loro soddisfazione deve essere perseguita dallo Stato al di sopra di qualsiasi altro interesse.

La mancanza di controllo della società sullo Stato, ancora dominata da interessi particolari, è indicata come un fattore negativo nella gestione pubblica. Per il Figlio (2005), la conservazione della sfera privata dellindividuo in concomitanza con la promozione dei desideri della comunità politica rappresenta il reale interesse pubblico che l'amministrazione deve perseguire.

Affinché l'efficienza migliori in modo significativo, non solo è necessaria la democratizzazione del sistema politico, ma anche l'inclusione di meccanismi democratici interni alla pubblica amministrazione, che sono legati alla formazione delle politiche pubbliche e alla Condivisione del potere nel processo decisionale.

I dirigenti pubblici, i cittadini e altri attori fanno parte di un sistema di governance dinamico, aperto e interattivo, in cui l'autorità dello Stato viene utilizzata in modi diversi per ottenere vari risultati pubblici.

In questo contesto, la partecipazione della società ha un valore incommensurabile, in quanto incoraggia la cittadinanza attiva. L'empowerment delle comunità e lo spirito 
civile possono fare la differenza nella gestione pubblica e, di conseguenza, nei risultati dei servizi pubblici forniti dallo Stato.

D'altra parte, il direttore pubblico dovrebbe riflettere le sue azioni, sotto l'egida del pensiero sistemico, il cui tema sarà discusso di seguito.

\section{PENSARE SISTEMICO}

Attraverso l'atto di pensare, gli esseri umani modellano il mondo, interagendo secondo i loro desideri, piani e obiettivi. I concetti e i processi di cognizione, sensibilità, coscienza e immaginazione (ANDRADE, 2016) possono essere associati al pensiero. Con un focus sull'evoluzione della scienza, possiamo citare i pensieri cartesiani predominanti, complessi e sistemici.

II pensiero sistemico è sorto nel XX secolo, opposto al pensiero riduzionistameccanicistico di Cartesio e Newton, che si basa sul paradigma scientifico tradizionale a cui fa riferimento Vasconcellos (2009), i cui presupposti di base sono riassunti in Semplicità, stabilità e obiettività.

L'assunzione della semplicità si basa sulla convinzione che sia necessario separare le parti per capire il tutto. In questo Look, Cunha (2006) interpreta che la riduzione è un'altra operazione alla ricerca della semplicità, che unifica ciò che è diverso. Trovando un fenomeno complesso, lo scienziato cerca la sua riduzione ad un altro fenomeno più semplice e già ben compreso.

L'ipotesi di stabilità risiede nella convinzione che ci sia stabilità nel mondo e che ci siano ripetizioni regolarmente. Sotto l'assunzione dell'obiettività, costituisce la convinzione che sia possibile conoscere il mondo come è composto in realtà, considerando il criterio essenziale del pensiero scientifico (CUNHA, 2006).

Il paradigma della scienza tradizionale, attraverso il pensiero lineare-cartesiano, si sviluppò e si stabilì nelle scienze fisiche ed esatte, diventando un modello di scientificità. I fisici hanno agito sulla base dei presupposti di semplicità, stabilità e obiettività, e ci sono riusciti, spiegando il mondo fisico e sviluppando tecnologie 
sofisticate che hanno modificato sempre più le relazioni dell'uomo con la natura (VASCONCELLOS, 2009).

Con le convinzioni che c'è una differenza tra gli esseri umani e la natura, e che i fenomeni umani sono di natura soggettiva, è stata stabilita la rottura tra la natura Scienze (oggettive) e scienze umane. Secondo Vasconcellos (2009), le scienze fisiche hanno facilmente adottato le tre ipotesi epistemologiche. Le scienze biologiche adottarono l'assunzione dell'obiettività e avevano problemi con quelle della semplicità e della stabilità, mentre le discipline umanistiche avevano difficoltà rispetto alle tre ipotesi, in particolare quelle dell'obiettività.

Il pensiero sistemico propone, al contrario, i paradigmi di complessità, instabilità e intersoggettività, che si integrano incredibilmente con la psicologia analitica di Carl Gustav Jung (VASCONCELLOS, 2009).

Un professionista che vive, vede il mondo e agisce in esso le implicazioni di aver assunto per se stesso i presupposti di complessità, instabilità e intersoggettività possono essere considerati un professionista sistemico (VASCONCELLOS, 2009).

II paradigma sistemico, che considera i presupposti di complessità, soggettività, instabilità, ha come principi la flessibilità e la plasticità che si manifestano nei sistemi attraverso Auto-organizativas caratteristiche che, dal caos, Presuppone aspetti dell'autorinnovamento e della trasparenza automatica. Così, sistemi aperti lontani dall'equilibrio possono preservare, riprodurre, rinnovare, adattarsi ed evolversi. Così, i processi di adattamento da una parte e dall'altra sono evidenti, quelli dell'apprendimento, dello sviluppo e dell'evoluzione (COSTA, 2007).

Cunha (2006) esprime la nozione che il pensiero sistemico significa pensare in termini di connessioni, relazioni, contesto, interazioni degli elementi di un tutto; Vedere le cose in termini di reti, reti e comunità.

II pensiero sistemico non nega la razionalità scientifica, ma crede di non fornire parametri sufficienti per lo sviluppo umano e per la descrizione dell'universo materiale. 
Per questo cerca di guardare nella direzione opposta del riduzionismo, dando maggiore enfasi al tutto che a parte.

Presuppone una metodologia dei processi di pensiero, che configurano l'esistenza del tutto. Cioè, è necessario studiare i sistemi a livello globale, al fine di coinvolgere tutte le loro interdipendenze, perché ciascuno degli elementi, quando assemblati per costituire un'unità funzionale più grande, sviluppare qualità che non sono nelle loro componenti Isolati (BERTALANFFY, 2009).

Capra (1996) sostiene che il pensiero frammentato non è in grado di affrontare l'interconnessione dei problemi globali, sia a livello superiore della società che a livello individuale. In questo senso, l'essenza del pensiero sistemico è nel cambiamento di mentalità, il che significa vedere le interrelazioni invece di creare catene lineari causaeffetto e vedere processi di cambiamento piuttosto che istantanee (Senge, 2009). In questa riunione, le modifiche non devono essere considerate come azioni isolate, ma come un insieme di misure interdipendenti che fanno parte della pianificazione dei miglioramenti dell'organizzazione.

È un modo di analizzare e pensare la conoscenza del mondo, attraverso i sistemi. Una visione sistemica o "pensiero sistemico" significa tenere a mente ciò che si vuole risolvere, scegliendo il modo giusto o più appropriato. Si può dire che questa è un'abilità che un individuo acquisisce per analizzare gli eventi e le loro possibili conseguenze, con l'obiettivo di creare una soluzione unica che contempla le aspettative di tutte le parti coinvolte.

Sia nella gestione privata che in quella pubblica, non c'è uniformità nell'adozione del pensiero sistemico da parte dei dirigenti. La maggior parte degli agenti pubblici non conosce nemmeno il concetto. Si può riaffermare l'importanza del pensiero sistemico nella gestione attraverso le parole di zanelli:

L'azione di somministrazione è legata alla pratica del pensiero sistemico e della leva strategica. Le caratteristiche della leadership, nel contesto dell'interazione umana che privilegia i processi di ottimizzazione dell'apprendimento, sono orientate all'esercizio della partecipazione a tutti i livelli e alla costruzione di squadre. 
Nel 2008, la National Quality Foundation (FNQ), presente nei più prestigiosi modelli mondiali di eccellenza, ha condotto un sondaggio virtuale con 196 dirigenti brasiliani. I risultati hanno rivelato che la maggior parte degli intervistati conosceva solo parzialmente il concetto di pensiero sistemico.

La grande preoccupazione degli scienziati sistemici è il riconoscimento che la necessità di un profondo cambiamento di percezione e di pensiero, per garantire la sopravvivenza, non ha ancora raggiunto la maggior parte dei dirigenti, né gli amministratori e gli insegnanti di Le nostre principali università (CAPRA, 1996).

Quando le persone percepiscono il mondo in modo diverso, emergono nuove credenze e presupposti, che consentono lo sviluppo di nuove competenze e capacità, formando un ciclo continuo di apprendimento organizzativo.

Peter Senge (2009) Luoghi che il pensiero sistemico può essere presentato come una disciplina, uno studio con teoria e pratica, che permette di sviluppare la visione dell'assemblaggio, una struttura per vedere le interrelazioni al posto delle cose, per vedere i modelli di cambiamento Al posto delle istantanee statiche. II manager, mentre adotta il pensiero sistemico, può fare la differenza nella gestione pubblica e cambiare la realtà del paese. Con questo nuovo look, è possibile condurre le azioni dell'istituzione, interpretando le politiche e le procedure e adattando i processi e le pratiche interne ai requisiti definiti dalla legge.

Ci vuole innovazione nella gestione pubblica. II manager deve assumere il ruolo e smette di agire come semplice supporto, svolgendo i compiti con coraggio. Pensare sistematicamente per il bene del benessere sociale.

Si tratta di un tema complesso, perché il cambiamento di postura del manager, nell'adottare il pensiero sistemico nell'esercizio della gestione, è legato a criteri soggettivi, come il carattere, la cui virtù non viene misurata o valutata quando l'ingresso di questo Professionista in attività pubblica, indipendentemente dal fatto che si trattasse di gara pubblica o di nomina per nomina. 
Tuttavia, come presentato nella ricerca, la gestione pubblica è in aumento del progresso, ampliando la capacità politica dello Stato di "fare bene la politica" per servire meglio la società e quindi raggiungere l'efficienza. Sempre più autori sostengono l'abbandono teorico del principio di supremazia. Gli interessi pubblici e gli interessi privati non sono chiaramente categorie antagonistiche, ma piuttosto complementari (JUSTEN FILHO, 2005).

La ricerca ci ha permesso di dedurre che l'applicazione dei concetti di pensiero sistemico può aggiungere un valore significativo a diverse aree di ricerca e nei settori delle organizzazioni. In questo senso, l'uso dell'approccio sistemico in altri settori può essere rivelato come un contributo accademico rilevante.

Pertanto, è valido affermare che l'obiettivo di questo studio è stato raggiunto, ed è possibile affermare che l'adozione del pensiero sistemico da parte del manager è direttamente correlata al principio di efficienza.

Per gli studi futuri, si propone di condurre ricerche negli organi della pubblica amministrazione, con l'obiettivo di verificare le caratteristiche comportamentali sistemiche dei manager, suggerendo pratiche alla gestione sistemica con particolare attenzione al comportamento delle persone .

\section{CONSIDERAZIONI FINALI}

Dopo aver analizzato i documenti trovati nella letteratura, è stato possibile identificare che l'adozione del pensiero sistemico da parte del manager può influenzare la direzione della gestione pubblica del paese. Si tratta di un'abilità che un individuo acquisisce per analizzare gli eventi e le loro possibili conseguenze, con l'obiettivo di creare una soluzione unica che contempla le aspettative di tutte le parti coinvolte. I benefici di questa nuova visione/postura possono essere visibili nei servizi resi alla società e nelle azioni interne dell'istituzione, perché è direttamente correlata al processo decisionale. 
A tal fine, il direttore pubblico, come nelliniziativa privata, ha bisogno delle caratteristiche e delle qualità che gli consentano di promuovere i cambiamenti proposti. In qualità di leader del settore pubblico, i dirigenti devono essere dotati di conoscenze, competenze e atteggiamenti, nonché di una forte vocazione ad affrontare le varie forme e fonti di potere che permeano il settore pubblico (MORGAN, 1996).

L'adozione del pensiero sistemico è intimamente legata allo sviluppo delle virtù etiche del manager. La visione del mondo è individuale. Presto, non c'è modo di avere un'unità o un controllo nel modo di pensare e di agire. In questa congiunzione, non dovrebbe essere considerato di cambiamenti come azioni isolate, ma come un insieme di misure indipendenti che fanno parte della pianificazione dei miglioramenti dell'organizzazione.

II pensiero sistemico "aiuta a capire come cambiare i sistemi in modo più efficace e come agire in migliore armonia con i processi del mondo naturale e sociale" (VALENA, 2011).

Le organizzazioni che avranno veramente successo in futuro saranno quelle che scopriranno come coltivare nelle persone l'impegno e la capacità di imparare a tutti $\mathrm{i}$ livelli dell'Organizzazione (SENGE, 2009).

Si deve pertanto considerare che il presente studio intende discutere una prospettiva ancora incipiente su un tema strategico per le agenzie pubbliche in termini di gestione, senza alcuna intenzione di esaurire l'argomento.

\section{RIFERIMENTI}

ABRUCIO, Fernando Luiz. Trajetória recente da gestão pública brasileira: um balanço crítico e a renovação da agenda de reformas. Edição Especial Comemorativa. Rio de Janeiro: Revista de Administração Pública (RAP), 2007.

ALVAREZ, Maria Esmeralda Ballestero. Organização, sistemas e métodos. São Paulo: McGraw-Hill, 1990. 
ALVES, João Bosco da Mota. Teoria Geral de Sistemas - Em busca da interdisciplinaridade. Florianópolis. Instituto Stela, 2012.

AMARAL, Antônio Carlos Cintra do. 0 princípio da eficiência no direito administrativo. Revista Diálogo Jurídico, Salvador, CAJ - Centro de Atualização Jurídica, n.14, jun./ago. 2002.

ANDRADE, A; Rodrigues, L; Seleme A; Souto, R; Pensamento sistêmico: Caderno de campo. $O$ desafio da mudança sustentada nas organizações e na sociedade. Porto Alegre: Bookman, 2006.

BINENBOJM, Gustavo. Da Supremacia do Interesse Público ao Dever de Proporcionalidade: Um novo paradigma para o Direito Administrativo. In: SARMENTO, Daniel (org.). Interesses públicos versus Interesses privados: desconstruindo o princípio da supremacia do interesse público. Rio de Janeiro: Lumen Juris, 2010.

BERTALANFFY, L. von. Teoria Geral dos Sistemas: Fundamentos, desenvolvimento e aplicações. São Paulo: Editora Fundação Perseu Abramo, 2006.

BRASIL. Constituição da republica Federativa do Brasil: 1988. 25. Ed. Brasília: Câmara dos Deputados, Coordenação de Publicações, 2012.

CÂMARA, Leonor Moreira. Fatores do Pensamento Sistêmico como Potencializadores de Sucesso de Projetos de Software no Setor Público.organização da administração pública Federal Brasileira: uma introdução ao estudo da organização da direção pública na perspectiva de estudos organizacionais. Rio de Janeiro: Revista de Administração Pública (RAP), 43(3), maio/junho 2009.

CAPRA, F. O Ponto de Mutação. A ciência, a sociedade e a cultura emergente. Trad. Álvaro Cabral, São Paulo: Cultrix, 2006.

CAPRA, Fritjof. A Teia da Vida. Uma Compreensão Cientifica dos Sistemas Vivos. São Paulo (SP). Editora Cultrix. 1997. 
CARNEIRO, R., MENICUCCI, TMG. Gestão pública no século XXI: as reformas pendentes. In Fundação Oswaldo Cruz. A saúde no Brasil em 2030 - prospecção estratégica do sistema de saúde brasileiro: desenvolvimento, Estado e políticas de saúde [online]. Rio de Janeiro: Fiocruz/lpea/Ministério da Saúde/Secretaria de Assuntos Estratégicos da Presidência da República, 2013. Vol. 1. pp. 135-194. ISBN 978-85-8110-015-9. AvailablefromSciELO Books.

CHIAVENATO, Idalberto. Recursos Humanos. O Capital Humano das Oganizações. SãoPaulo:Atlas, 2004.

CHIAVENATO, Idalberto. Iniciação à Organizações e Controle.São Paulo: McGrawHill, 1989.

COSTA, WedjaJosefa Granja. Socionomia de base sistêmica: Método de apoio à gestão de grupos na organização. Dissertação de Mestrado. Fortaleza: Universidade Federal do Ceará, 2007.

CUNHA, Aura Celeste Santana. Pensamento Sistêmico e tecnologia educacional: a metodologia WEBQUEST. Dissertação de Mestrado Profissional em Computação. Fortaleza: Universidade Estadual do Ceará, 2006.

DRUCKER, Peter. The effective executive. Harper Collins Publishers, 1993.

DRUCKER, Peter F. Administrando para o Futuro: os anos 90 e a virada do século. Tradução de Nivaldo Montigelli Jr. São Paulo: Pioneira Thomson Learning, 2002.

FALCONI, Vicente. O verdadeiro Poder.Nova Lima: INDG Tecnologia e Serviços Ltda. 2009.

FAYOL, Henri. Administração Industrial e Geral.São Paulo. Atlas, 1990.

FLEURY, S. J. F. Redes de proteção: incentivos, escolhas e comportamentos na política brasileira. 2009. Tese (Doutorado) - Faculdade de Filosofia e Ciências Humanas da Universidade Federal de Minas Gerais, Belo Horizonte, 2009. 
HERNES, T. Four ideal-types organizational responses to the new public management reforms and some consequences. International Review of Administrative Sciences, v. 71, n. 1, p. 5-17, 2005.

HOOD, C. A public management for all seasons? Public Administration, . 69, $\mathrm{n}$. $1,1991$.

JUSTEN FILHO, Marçal. Curso de Direito Administrativo. São Paulo: Saraiva, 2005.

GOMES, Lauren Beltrão et al . As origens do pensamento sistêmico: das partes para o todo. Pensando familiar, Porto Alegre, 2014.

KOLTER, John P. Liderando Mudanças. Rio de Janeiro: Elsevier, 2013.

LAKATOS, Eva Maria; MARCONI, Mariana de Andrade. Fundamentos de Metodologia Científica. 4 ed. São Paulo: Atlas, 2001.

LIMA, Joilson Souza de. O Planejamento Estratégico como Ferramenta de Gestão. Revista Científica Multidisciplinar Núcleo do Conhecimento. Ano 03, Ed. 03, Vol. 03, pp. 58-69, Março de 2018. ISSN:2448-0959.

MEIRELLES, Hely Lopes. Direito Administrativo Brasileiro. São Paulo: Malheiros, 2002.

MANNING, N. et al. Reformas de gestão pública: o que a América Latina tem a aprender com a OCDE.In: MEDEIROS, P. C.; LEVY, E. (Orgs.). Novos caminhos da gestão pública: olhares e dilemas. Rio de Janeiro: Qualitymark; Brasília: CONSAD, 2009. p. 97-148.

MORAES, Germana de Oliveira. Controle Jurisdicional da Administração Pública. São Paulo: Dialética, 1999, p. 127.

MORGAN, Goreth. Imagens da Organização. São Paulo: Atlas, 1996. 
PIETRO, Maria Sylvia Zanella Di. Direito Administrativo. São Paulo: Atlas, 2002.

SENGE, Peter M. A quinta disciplina: arte e prática da organização que aprende. $25^{\mathrm{a}}$ ed. Rio de Janeiro: BestSeller, 2009.

SEMLER, Ricardo. Virando a própria Mesa: Uma história de Sucesso Empresarial. Rio de Janeiro, 2002.

VALENÇA, Antônio Carlos. Aprendizagem Organizacional: 123 aplicações práticas de arqueótipos sistêmicos. São Paulo:SENAC, 2011.

VASCONCELLOS, Maria José Esteves. Pensamento Sistêmico: O novo paradigma da ciência. 9aㅗ ed. Campinas-SP: Papirus,2010.

ZANELLI, José Carlos. Interação Humana e Gestão: a construção psicossocial das organizações de trabalho. São Paulo: Casa do Psicólogo, 2008.

Inviato: Ottobre, 2019.

Approvato: ottobre 2019. 